\title{
Medicine and mental illness: how can the obstacles sick doctors face be overcome? ${ }^{\dagger}$
}

\author{
Anonymous ${ }^{1}$
}

The Psychiatrist (2012), 36, 104-107, doi: 10.1192/pb.bp.111.034629

${ }^{1}$ The author of this article wishes to remain anonymous

Correspondence to The Psychiatrist (tp@rcpsych.ac.uk)

First received 16 Mar 2011, final revision 17 Jun 2011, accepted 6 Jul 2011

\begin{abstract}
Summary One in four of the UK population is expected to have a mental health problem at some time in their lives. There is no reason to believe that this statistic should not apply to doctors just as it does to everyone else. This paper discusses the obstacles to accessing treatment that doctors with mental ill health face, and goes on to consider potential solutions to these problems. There is a great pool of talent within the field of medicine and those doctors with mental health problems often have increased empathy as well as professional expertise. This article sets out to demonstrate that there are ways of supporting these doctors and enabling them to overcome their illnesses and regain satisfaction in their work.
\end{abstract}

Declaration of interest The author has schizoaffective disorder and has received treatment while a doctor.
Public awareness campaigns have made it a widely known fact that one in four people will have a mental illness at some point in their lives; however, in my experience, we as doctors do not tend to apply this statistic to ourselves. Psychiatrists may come across some of the $25 \%$ of doctors who have mental health problems; there will be many others who suffer in silence, unable or unwilling to seek help. This is worrying because doctors who do not get appropriate treatment for their mental illness may become unsafe in their working practice, and have, in some cases, taken their own life because of their distress. It is clearly important that psychiatrists, as well as general practitioners (GPs) and accident and emergency (A\&E) doctors, think about how best they can help doctors who are psychiatrically unwell. In this paper, I discuss the obstacles that doctors face in accessing treatment, and the particular needs that they have when they do seek help. I will also suggest ways in which psychiatrists, together with colleagues in other specialties, can improve the treatment experience for mentally ill doctors, increasing their chances of rehabilitation.

\section{Background}

In writing this paper, I will draw on my own experience of being a doctor with mental illness to give examples of good practice and areas where in my case treatment could have been improved. I first attended a psychiatrist in my final year at medical school. I graduated but have since spent considerable time in hospital and have been unable to work. I have been variously diagnosed with major depressive disorder, atypical eating disorder, borderline personality

†See editorial, pp. 81-84, this issue. disorder and bipolar disorder; however, my condition has evolved and I am currently being treated for schizoaffective disorder. It is my hope that one day - with the right treatment - I will regain fitness to practise.

\section{Obstacles faced by doctors in accessing treatment}

A buzzword in all mental health campaigns is 'stigma'. There has been a lot of work done to de-stigmatise mental illness in the community; however, in my experience, stigma remains a barrier to doctors accessing treatment. One reason for this is that there remains a culture within medicine that doctors must be personally strong and of a sound mind. Another is that seeing a colleague become mentally ill can make doctors feel uncomfortable as they recognise their own vulnerabilities. Because of this stigma, doctors may decide not to seek expert help as they fear that their colleagues will find out about their problems and treat them differently. I certainly found that doctors who had previously been my friends changed in their attitude towards me after I had been detained under the Mental Health Act, and particularly when I started to struggle with self-harm. In fact, it was my non-medical friends who stuck by me through the most difficult times, which I think bears out the theory that mental illness in a colleague is somehow 'too close to home'. Conversely, when I have presented with physical disease, treating doctors have been relaxed in their approach and have apparently enjoyed talking to me as a doctor with a specific problem rather than as a problem in myself.

Another obstacle to accessing care is that doctors may fear being treated by their own colleagues. This could be an issue for someone presenting to $\mathrm{A} \& \mathrm{E}$, for example, but is 
clearly a particular concern within the specialty of psychiatry. Doctors may feel ashamed of their mental illness as a sign of personal frailty, and they may fear that confidentiality may not always be kept, knowing that treating doctors do tend to discuss cases with colleagues, whether socially or to seek advice. In my case, I became aware during a hospital admission that the team looking after me had discussed me with doctors whom I knew and who were not involved in my care. It is crucial that maintaining confidentiality is not just considered to mean withholding information from those outside the medical profession, but also that personal information is kept within the treatment team. There may be a case for allowing doctors to be treated under another name; there is certainly a case for improving medical education so that students understand that discussing a doctor's case is unacceptable.

In relation to emergency treatment, there was a period in my life when I was self-harming regularly and also attempted suicide on a number of occasions. I was forced to attend A\&E frequently and was often seen by former colleagues who were at foundation year 2 (F2) level at that time. Some were indeed kind and treated me like any other patient, which put me at ease and helped me to feel less like I was in some way aberrant; others visibly recoiled from me, unable to make eye contact. There were even those who expressed anger and frustration with me; 'Why are you doing this to yourself?'. One doctor did not speak to me at all, stapling a wound without washing it. Needless to say, an infection set in within 2 days that left me quite seriously ill and back in A\&E. In fact, the reality was that I received better treatment when I did not know the doctor and did not divulge my own profession. True, I was patronised at times, but I preferred that to being judged.

A crucial reason that many doctors in the UK fear admitting to mental health difficulties is that they are concerned about having to disclose their problems to the General Medical Council (GMC). I was ultimately declared unfit to practise, due to the unpredictability of my illness and my episodes of psychosis; however, most mental health problems that doctors experience do not result in this outcome. What I learnt, and what is important to note, is that the GMC would much prefer to be aware of problems that a doctor is facing and to know that they are accessing the appropriate treatment than to think that a sick doctor was continuing to practice without informing them or receiving treatment for their condition. In the latter case, fitness to practise is much more likely to be called into question once the reality of the situation emerges.

On a practical level, waiting lists can be a barrier to doctors who do wish to access services, and it has become more difficult for doctors in any specialty to offer colleagues rapid assessment as managers impose protocol from above. I was once offered psychotherapy but was told that I would have to wait 18 months for treatment. At that time, I took the decision not to join the waiting list as I sincerely hoped that I would be better by then and did not see any point.

There is also an issue with regard to referral to psychiatric services. Many doctors who present to their GP with a mental health issue will play it down, for example because of their worries about the GMC, and the GP will opt to manage the illness themselves initially. Some doctors may present with fatigue or another somatic symptom, and GPs may fail to screen for underlying depression. In either case, referral will be delayed until the doctor's mental health has deteriorated significantly. I was fortunate in that my GP picked up early that my low mood was significant and referred me when I first presented, but this does not always happen.

Aside from fears around admitting to mental health problems and practical obstacles, there is a genuine issue where the attitudes of healthcare staff in general are concerned. These may have a role in deterring doctors from accessing the correct help. I found that very often when I presented to A\&E or the out-of-hours GP services, nurses and other staff would make comments like, 'But you're an intelligent girl, why would you do this to yourself?' or 'You ought to know how to deal with this'. Evidently, they failed to comprehend that mental illness does not discriminate on the basis of intelligence; indeed, in my experience, being intelligent has made my experience of illness all the more difficult.

When I was self-harming, I also regularly encountered staff who told me that there were 'actual sick people' who needed attention. In spite of my distress, I was not considered to be ill; instead, I was made to feel that I was a problem and a nuisance at a time when my self-esteem was already at its lowest. It was clear to me that some A\&E staff had little understanding of mental illness, and even community psychiatric nurses who 'risk assessed' me before I was discharged seemed somehow intimidated by me because of my degree, even though I was in a vulnerable position. Because of this, they often came across as hostile and aloof when what I needed was warmth and compassion.

\section{What can be done to help doctors with mental illness access appropriate care?}

All of these factors beg the question: what can we do to help doctors with mental health problems access appropriate services? First of all, where stigma is concerned, it would be helpful to have a campaign specifically directed at doctors to remind them that one in four of them will experience mental distress. Ideally, doctors who have had a mental illness would speak out, becoming role models whose example others could follow, a model which has worked in general public awareness drives. One possible aim of such a campaign would be to sensitise doctors to the potential problems that their colleagues may experience, so that they can be alert to difficulties and encourage people to seek help. A similar campaign was recently directed at politicians, with one outcome being that the rule against people who have been detained under the Mental Health Act becoming members of Parliament was removed from law. This shows that targeted campaigning can be successful when planned effectively.

In addition to an anti-stigma campaign, there is a need for a widespread educational effort to improve understanding about mental illnesses among all health professionals. Negative attitudes to someone who has self-harmed, for example, should become unacceptable as a culture of compassion is fostered towards all those who have psychological problems, whether they are considered to be mentally ill or to have a disorder of personality. 
Personality disorders deserve particular mention because there remains - even within psychiatry - a greater degree of stigma and a lesser degree of understanding than there is with conditions such as depression.

Where treatment by colleagues is concerned, this should be avoided as far as possible. In my opinion, doctors with mental health problems should have early input from a consultant psychiatrist and if they present to A\&E should be treated by the most senior doctor on duty. On one occasion, I was treated for an overdose by a trainee but received a follow-up telephone call from an emergency medicine consultant the day after discharge. At the time, this meant a lot to me, as it showed that someone senior was aware and cared about my continuing well-being. On the other hand, where a colleague must treat the sick doctor, a culture of professionalism should prevail. I believe that there should also be a mechanism whereby access to mental health services can be fast-tracked for doctors. It can be argued that doctors should not have preferential treatment within the National Health Service (NHS), but the fact is that fewer patients may have to wait for treatment if a sick doctor gets help quickly and can get back to work sooner or have the capacity to work more effectively.

In my experience, having access to brief in-patient stays has been helpful in imposing rest and allowing me to 'be unwell' rather than maintaining a semblance of health, and has led to a quicker recovery. I believe that doctors can be treated in general acute units; however, they should generally be advised not to reveal their profession to other patients and should be supported in their anonymity. Treating doctors in a national specialist service would remove them from home support symptoms and make reintegration difficult, and could paradoxically increase the level of stigma.

On a similar theme, GPs should be encouraged to screen for mental health issues among their medically qualified patients, and to refer early if they suspect that there is a problem. A GP can be a key support person in a sick doctor's network. For example, my GP has often seen me on a weekly basis to check in with me and make sure that I am coping with any changes in medication or mood. The fact that my GP continues to take the initiative in making me review appointments reassures me that I am not overusing her service and the continuity of care that I receive offers me a sense of security during difficult periods. There is an agreement within my practice that I will be seen by partners only, which prevents me from being offered appointments with trainees of my own age and level. In the GP setting as in secondary care, I find it helpful that I am treated as a doctor as well as a patient, and the fact that this has continued in spite of my failure to be deemed fit to practise boosts my self-esteem. It has also been helpful that I have a primary care plan so that my GP can increase my medication quickly without having to wait for psychiatric approval. This has allowed me to recover from episodes of illness more rapidly.

General practitioners and psychiatrists alike should be aware that the involvement of allied health professionals in a doctor's care can be extremely helpful; there is no reason why doctors should only be treated by doctors. I have found consistent input from a social worker who brings an outside perspective and is a little removed from my former life as a doctor very beneficial. Similarly, talking on a weekly basis with a non-medically qualified but accredited counsellor about my struggles as a doctor-patient helps me to process them and to feel more in control of my life. Her office is a 'safe place' where I know confidentiality - within standard boundaries - will be maintained, and I am less intimidated by talking with her than I am by talking with a medical professional.

The GMC question is clearly one that looms large in the minds of most doctors who feel they may be struggling mentally. It is imperative that clear guidelines are issued regarding when a condition needs to be declared and the consequence if one fails to do so. Doctors who are given adequate support through the process of approaching the GMC, for example by a mentor or their consultant psychiatrist, are likely to cope better with this process than those who feel they are doing it on their own. Clearly, there may be instances when a doctor has concerns about the mental health of a colleague. In my opinion, the GMC should only be informed by a third party if there are concerns about patient care and the doctor concerned has been approached first. Doctors should be aware that the role of the GMC is to protect patients and that fitness to practise procedures are long and arduous; they should therefore only be involved when absolutely necessary.

In my experience, when they do present for treatment, sick doctors benefit from being given the same information about their condition and treatment from health professionals as any other patient. Knowledge should not be assumed, although doctors have the same right to be involved in decisions about their treatment as anyone else and ought to be given the opportunity to express concerns about a particular treatment plan. Any rehabilitation plan should be tailored to the sick doctor's level of intelligence. For example, I was once assigned to an occupational therapy class where the chief activity was colouring pictures in children's drawing books. I eventually told the occupational therapist that being reduced to this was making me more depressed, and she was able then to find a correspondence course that I could work on while the others coloured and drew.

Other practical measures that may help doctors with mental health problems include ensuring that adequate sick leave is facilitated in order to promote recovery. Locum cover should be provided so that the sick doctor is not left feeling as if they are causing a problem or creating a heavier workload for colleagues. When the sick doctor is ready to go back to work, provision should always be available for a phased return where the hours are only increased as the doctor's health and stamina improve. A mentor could be appointed within the clinical environment who could check that the doctor was coping and provide support if necessary. These practices are common within other professions and GPs will be familiar with 'fit notes' that give the option of recommending adjustments or returning part time initially. Employers are obliged to facilitate reasonable adjustments and there is no reason why the NHS should be an exception. 


\section{Conclusion}

Given the numbers of doctors likely to be have some degree of mental ill health, it is important that psychiatrists and other mental health professionals are aware of the obstacles that these doctors may face in seeking help, and that they actively partake in activities that make the process easier and more acceptable. I have described how stigma, issues around treatment by colleagues, practical difficulties, fear of implications for fitness to practise and poor attitudes and understanding can prevent sick doctors from getting the care they need. I have gone on to offer certain suggestions for how some of these obstacles can be overcome. Even in the twenty-first century, it is not easy for anyone to admit to having a mental health problem. This must change, and we, as doctors, should be leading the way in making stigma a thing of the past.

\section{About the author}

The author has requested to remain anonymous but is now a freelance writer and has not self-harmed for 3 years.

\title{
Going the extra mile ${ }^{\dagger}$
}

\author{
Chris Fear, ${ }^{1}$ Mark Scheepers, ${ }^{1}$ Martin Ansell, ${ }^{1}$ Rosemary Richards, ${ }^{1}$ Paul Winterbottom ${ }^{1}$
}

The Psychiatrist (2012), 36, 107-108, doi: 10.1192/pb.bp.112.038679

${ }^{1}$ The ${ }^{2}$ gether NHS Foundation Trust, Gloucester, UK

Correspondence to Chris Fear (chris.fear@glos.nhs.uk)

First received 20 Jan 2012, accepted 20 Jan 2012
Summary Stephen Tyrer's commentary on Fair Horizons was generally supportive of the principles underpinning the model, while offering a number of questions and caveats. In this response, we provide responses to the points made and offer further insights into the practicalities of the Fair Horizons model.

\section{Declaration of interest None.}

We are grateful for the opportunity to respond to Professor Tyrer's commentary, ${ }^{1}$ which is both balanced and considered. Recognising the potential benefits of the Fair Horizons model at both patient and organisational levels, he raised the entirely valid point that we lack, as yet, data to support the approach, although we are at an advanced stage of implementation and thus committed, as an organisation, to its success. We would like to provide a brief response to the specific questions raised in the commentary.

Since the concept was developed, over 5 years ago, Fair Horizons has been honed through an iterative process of clinical engagement on the basis of a number of unarguable principles: that services must be clinically driven, equitable, person-centred and non-discriminatory, and that they must include prevention of mental ill health and promotion of well-being and recovery. Further, there is a commitment to early intervention across the spectrum of mental disorders, engagement of service users and carers, and quality and best practice. These principles were tested through consultation events with clinicians, service users, carers and commissioners, and have had a $97 \%$ positive acceptance and a willingness to engage with the change process.

'This is the authors' response to Stephen Tyrer's commentary ${ }^{1}$ on their original paper, Psychiatrist 2012; 36: 25-30.
Although acknowledging the 'sound theoretical underpinning' of the service model, Professor Tyrer has voiced concerns about its requiring significant commitment from all staff. Staff engagement with the process of change is part of an externally funded research project in collaboration with Queen Margaret University, Edinburgh, using the Flight Gate practice development tool. ${ }^{2}$

It is likely that our paper was insufficiently detailed to indicate that the first-point-of-contact centre has clinician support for the administrative function. The current process of allocating referrals is largely administrative within teams but may differ according to the culture of that team. Providing a central pathway avoids idiosyncrasies of culture and overcomes boundaries so that patients are less likely to become lost or to 'bounce' from team to referrer. Under Fair Horizons, administrative staff complete the initial information gathering and follow an algorithm, but this process is overseen by a clinician, with access to consultant psychiatric input for complicated cases.

We do acknowledge the concerns about Improving Access to Psychological Therapies, but consider this to be a national priority, with locally agreed, population-based figures outlining the wider service. Specialist psychological therapies continue to be an integral part of clinical services within Fair Horizons.

In the financial year 2010/2011, the trust received about 11000 referrals, of which 380 were for people with an 